\title{
NEU - Hands On Workshops - NEU
}

Jetzt anmelden (www.oerg.at)

\section{Donnerstag, 24. Oktober 2013}

$\nabla$

\author{
WS 1 CT-Protokolle made easy \\ 13:00-15:00 \\ Ein interaktiver Workshop \\ Koordinator/in: E. Sorantin, Graz \\ S. Weissensteiner, Graz \\ (3 DFP Punkte)
}

Der Workshop „CT- Protokolle made easy - ein interaktiver Workshop“ soll Kenntnisse und Fertigkeiten vermitteln um CT Protokolle an die verschiedensten Fragestellungen und Altersgruppen, insbesondere Kinder, dosissparend anzupassen.

\section{WS 2 Muskuloskelettaler Ultraschall 13:00-17:00}

Hands-on WS: Schulter \& periphere Nerven der oberen Extremität Koordinatorin: A. Klauser, Innsbruck (6 DFP Punkte)
In Abstimmung zum diesjährigen Thema der Sportverletzungen der oberen Extremität möchten wir in diesem Hands-On Kurs ebenso die Sonografie der oberen Extremität anbieten. So empfiehlt es sich diese standardisiert (ESSR Guidelines: www.essr.org) zu untersuchen, wodurch eine Vielzahl von diagnostischen Fragestellungen bereits beantwortet werden kann.

\section{WS 3 Periphere Gefäßinterventionen 13:00-17:00}

Simulatortraining

Koordinator: K. A. Hausegger, Klagenfurt (6 DFP Punkte)

Tutoren: Herbert Langenberger, Michael Hauser

Die Workshopteilnehmer(innen) haben die Gelegenheit in Kleingruppen zu jeweils maximal 5 Personen unterschiedlichste transarterielle periphere Gefäßinterventionen an" State-of-the-art" Simu- latorsystemen durchzuführen. An jedem Simulator werden die WS-Teilnehmer(innen) von einem erfahrenen interventionellen Radiologen, der als Tutor tätig ist, unterstützt.

\section{WS 4 „Tomosynthese“ - in Vorberei- tung Details folgen in Kürze}

Genauere Informationen zu den einzelnen Workshops finden Sie auf www.oerg. at/oerg2013

Eine Anmeldung zu den Workshops ist nur in Kombination mit einer Kongressregistratur möglich. Die Kurskosten betragen $€ 50,00$.

ACHTUNG: beschränkte Teilnehmerzahl!

Die Anmeldung erfolgt nach dem Prinzip „first come, first served“. 